\title{
Электронные и оптические свойства тонких пленок GaAlAs/GaAs
}

\author{
() Б.Е. Умирзаков, С.Б. Донаев, Н.М. Мустафраева \\ Ташкентский государственный технический университет им. И.А. Каримова, \\ 100095 Ташкент, Узбекистан \\ e-mail: sardor.donaev@gmail.com
}

Поступило в Редакцию 9 сентября 2017 г. В окончательной редакции 16 марта 2018 г. Принято к публикации 1 марта 2019 г.

Показано, что формирование на поверхности GaAs нанопленок GaAlAs приводит к увеличению значения коэффициента эмиссии истинно-вторичных электронов и квантового выхода фотоэлектронов, что объясняется отличием глубины зоны выхода истинно-вторичных электронов для GaAs и для GaAlAs.

Ключевые слова: эмиссионные свойства, оптические свойства, нанопленка, ионная имплантация, пленки $\mathrm{GaAs}$, нанокристаллические фазы.

DOI: 10.21883/JTF.2019.10.48177.2475

\section{Введение}

В настоящее время хорошо изучены состав, структура, оптические и электронные свойства многослойных структур $\mathrm{Ga}_{1-x} \mathrm{Al}_{x} \mathrm{As} / \mathrm{GaAs}$, полученных различными методами эпитаксии, что связано с широким использованием их в различных приборах микро- и оптоэлектроники [1-6]. Постоянные решетки $\mathrm{Ga}_{1-x} \mathrm{Al}_{x} \mathrm{As}$ и GaAs хорошо согласуются друг с другом, а их ширины запрещенных зон отличаются в $\sim 1.5$ раза. Авторами ранее [7] исследованы морфология и электронные свойства поверхности нанокристаллических фаз и нанопленок $\mathrm{Ga}_{0.5} \mathrm{Al}_{0.5} \mathrm{As}$ с толщиной $2.0-7.0 \mathrm{~nm}$, созданных на поверхности $\mathrm{GaAs}(111)$ имплантацией ионов $\mathrm{Al}^{+}$в сочетании с отжигом. Показано, что ширина запрещенной зоны $E_{g}$ нанопленок $\mathrm{Ga}_{0.5} \mathrm{Al}_{0.5} \mathrm{As}$ составляет $\sim 2.1-2.3 \mathrm{eV}$, а для нанокристаллов - $2.9 \mathrm{eV}$. Однако до настоящего времени практически не изучены эмиссионные и оптические свойства тонких $(d \leq 10 \mathrm{~nm})$ пленок GaAlAs/GaAs. Подобные исследования дают богатую информацию об электронных свойствах и кристаллической структуре исследуемых пленок.

Настоящая работа посвящена изучению энергетических и угловых зависимостей коэффициентов вторичной электронной эмиссии (ВЭЭ), коэффициентов отражения и поглощения света пленкой $\mathrm{Ga}_{0.5} \mathrm{Al}_{0.5} \mathrm{As} / \mathrm{GaAs}$.

\section{Методика эксперимента}

В качестве объектов исследования были выбраны монокристаллические образцы $p$-типа $\mathrm{GaAs}(111)$ с размерами $10 \times 10 \times 0.8 \mathrm{~mm}$. Основные исследования проводились для пленок $\mathrm{Ga}_{0.5} \mathrm{Al}_{0.5} \mathrm{As} / \mathrm{GaAs}(110)$ с толщиной $\sim 5-10 \mathrm{~nm}$, полученных имплантацией ионов $\mathrm{Al}^{+}$в GaAs с энергиями $1-8 \mathrm{keV}$ при дозе $D \approx 8 \cdot 10^{16} \mathrm{~cm}^{-2}$ в сочетании с прогревом при $T=850-900 \mathrm{~K}$ (образцы № 1) [7]. Известно, что незначительное изменение состава или параметров решетки пленки и подложки может привести к существенному изменению свойства гетероструктуры [6-9]. Дальнейшее увеличение энергии ионов приводило к увеличению коэффициента распыления поверхности и уменьшению концентрации $\mathrm{Al}$ в области максимума. Следовательно, этим методом получить пленки $\mathrm{Ga}_{0.5} \mathrm{Al}_{0.5} \mathrm{As}$ с толщиной $>10 \mathrm{~nm}$ c хорошей стехиометрией было невозможно. Поэтому одновременно были исследованы свойства пленок $\mathrm{Ga}_{0.5} \mathrm{Al}_{0.5} \mathrm{As} / \mathrm{GaAs}(111)$ с толщиной 5-20 nm, полученных методом молекулярно-лучевой эпитаксии (МЛЭ) (образцы № 2).

Исследования проводились снятием зависимостей $\delta\left(E_{p}\right)$ и $\eta\left(E_{p}\right)$ в широкой области энергии первичных электронов $\left(E_{p} \approx 5-1000 \mathrm{eV}\right)$ и угловых зависимостей $\delta(\varphi)$ и $\eta(\varphi)$, где $\delta-$ коэффициент истинновторичных электронов, $\eta$ - коэффициент неупругоотраженных электронов, измерения интенсивности проходящего света через образец, а также измерение коэффициентов преломления и отражения света. Зонноэнергетические параметры определялись по данным ультрафиолетовой фотоэлектронной спектроскопии и спектроскопии упруго отраженных электронов. Состав поверхности контролировался методом оже-электронной спектроскопии (ОЭС), а профили распределения атомов по глубине исследовались методом ОЭС в сочетании с распылением поверхности пленки ионами $\mathrm{Ar}^{+}$c энергией $3 \mathrm{keV}$ при угле падения $85^{\circ}$ относительно нормали. Методика эксперимента более подробно описана в [10]. При снятии угловых зависимостей $\delta(\varphi)$ и $\eta(\varphi)$ мишень поворачивалась относительно пучка первичных электронов на $\pm 70^{\circ}$. Во всех остальных случаях пучок электронов направлен на поверхность мишени перпендикулярно.

\section{Экспериментальные результаты и их обсуждения}

В таблице приведены значения термо- и фотоэлектронной работы выхода (е $\varphi$ и еФ), максимальное 
Основные эмиссионные характеристики $\mathrm{GaAs}$ ( $p$-тип) и пленки GaAlAs

\begin{tabular}{c|l|c|c|c|c|c|c}
\hline $\begin{array}{c}\text { Исследуемый } \\
\text { объект }\end{array}$ & $\begin{array}{c}\mathrm{e} \varphi, \\
\mathrm{eV}\end{array}$ & $\begin{array}{c}\mathrm{e} \Phi \\
\mathrm{eV}\end{array}$ & $\begin{array}{c}E_{g}, \\
\mathrm{eV}\end{array}$ & $\begin{array}{c}E_{p m}, \\
\mathrm{eV}\end{array}$ & $\delta_{m}$ & $\mathrm{H}$ & $\begin{array}{c}K, \\
(h v=10.8 \mathrm{eV})\end{array}$ \\
\hline $\mathrm{GaAs}$ & 5 & 5.1 & 1.4 & 500 & 1.1 & 0.26 & $3 \cdot 10^{-3}$ \\
$\mathrm{Ga}_{0.5} \mathrm{Al}_{0.5} \mathrm{As}$ & 5.1 & 5.3 & 2.1 & 550 & 1.4 & 0.25 & $6 \cdot 10^{-3}$
\end{tabular}

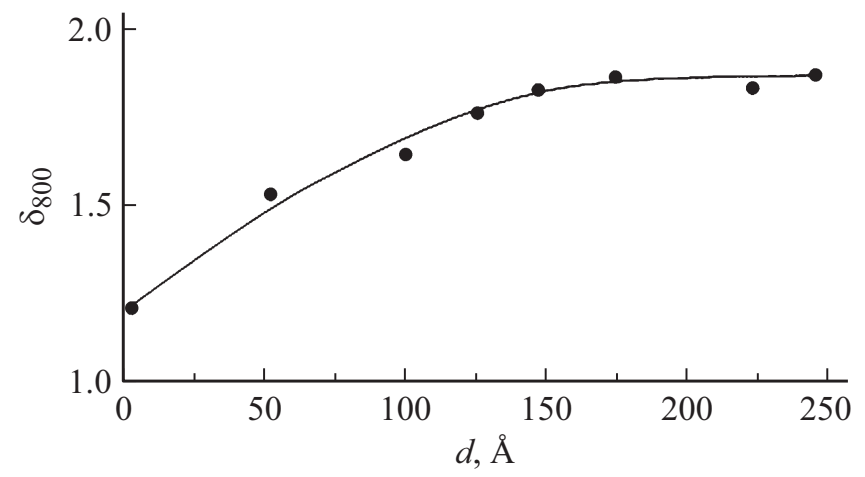

Рис. 1. Зависимость значения $\delta$ при $E_{p} \approx 800 \mathrm{eV}$ от толщины $d$ МЛЭ-пленки $\mathrm{Ga}_{0.5} \mathrm{Al}_{0.5} \mathrm{As} / \mathrm{GaAs}$.

значение коэффициента истинно-вторичных электронов (КИВЭ) $\delta_{m}$ и соответствующей ему энергии первичных электронов $E_{p m}$, значение коэффициента неупруго отраженных электронов при $E_{p} \approx 800 \mathrm{eV} \mathrm{H}$, а также значение квантового выхода фотоэлектронов $K$, снятого при $h v=10.8 \mathrm{eV}$ для чистого монокристалла $\mathrm{GaAs}(111)$ и $\mathrm{GaAs}$ с пленкой $\mathrm{Ga}_{0.5} \mathrm{Al}_{0.5} \mathrm{As}$ толщиной $\sim 50 \AA$ (образец № 1).

Из таблицы видно, что в случае GaAs c нанопленкой $\mathrm{Ga}_{0.5} \mathrm{Al}_{0.5} \mathrm{As}$, несмотря на некоторое увеличение е $\varphi$, значения $\delta_{m}$ и $K$ заметно увеличиваются. Отметим, что плотность (в $\left.\mathrm{g} / \mathrm{cm}^{3}\right) \mathrm{Ga}_{0.5} \mathrm{Al}_{0.5} \mathrm{As}$ на $\sim 20 \%$ меньше, чем плотность GaAs. Однако плотность атомов (число атомов в $1 \mathrm{~cm}^{3}$ ) в GaAs и GaAlAs практически идентичны, и ионы элементов $\mathrm{Ga}$ и $\mathrm{Al}$ имеют почти одинаковые размеры. Поэтому $\eta_{\mathrm{GaAs}} \approx \eta_{\text {GaAlAs }}$ и, следовательно, эмиссионные эффективности слоев GaAs и GaAlAs мало отличаются друг от друга. Тогда увеличение $\delta_{m}$ и $K$ в основном могут быть обусловлены увеличением ширины запрещенной зоны и глубины зоны выхода $\lambda$ истинно-вторичных электронов $\delta$. В настоящей работе $\lambda$ определялась методом снятия зависимости $\delta_{800}(d)$ [11], где $\delta_{800}-$ значения $\delta$ при $E_{p}=800 \mathrm{eV}, d-$ толщина пленки $\mathrm{Ga}_{0.5} \mathrm{Al}_{0.5} \mathrm{As}$, выращенной на поверхности $\mathrm{GaAs}$ методом МЛЭ. Величина $\lambda$ для чистого GaAs определялась предварительно и составила $\sim 100-120 \AA$. На рис. 1 приведена зависимость $\delta_{800}(d)$ для системы $\mathrm{Ga}_{0.5} \mathrm{Al}_{0.5} \mathrm{As} / \mathrm{GaAs}$.

Видно, что значение $\delta_{800}$ при $d \geq 150-160 \AA$ с ростом $d$ практически не меняется, поэтому можно полагать, что глубина зоны выхода ИВЭ (а также фотоэлектронов) $\lambda$ для $\mathrm{Ga}_{0.5} \mathrm{Al}_{0.5} \mathrm{As}$ составляет $150-160 \AA$.
В гетероструктурных системах степень кристалличности и эпитаксиальности нанопленки представляет особый интерес. При $d<\lambda$ степень эпитаксиальности можно оценить по угловым зависимостям коэффициентов ВЭЭ. На рис. 2 приведены зависимости $\delta_{800}(\varphi)$ для чистого $\mathrm{GaAs}(111)$ и для $\mathrm{GaAs}$ с пленкой $\mathrm{Ga}_{0.5} \mathrm{Al}_{0.5} \mathrm{As}$ c $d \approx 50 \AA$, полученные двумя способами: ионной имплантацией с сочетанием отжига и МЛЭ. Угол $\varphi$ определялся относительно нормали образца.

Видно, что во всех случаях с ростом $\varphi$ происходит немонотонный рост значения $\delta_{800}$, т.е. на зависимости $\delta_{800}(\varphi)$ наблюдаются максимумы и минимумы, положения которых соответствуют определенным кристаллографическим направлениям [12]. Угловые положения максимумов GaAs и GaAlAs, полученные методом ионной бомбардировки, хорошо совпадают друг с другом. Поэтому можно сделать вывод, что в данном случае происходит строгий эпитаксиальный рост пленки $\mathrm{Ga}_{0.5} \mathrm{Al}_{0.5} \mathrm{As}$. В случае МЛЭ-пленки $\mathrm{Ga}_{0.5} \mathrm{Al}_{0.5} \mathrm{As}$ на зависимости $\delta_{800}(\varphi)$ интенсивности пиков, характерных для $\mathrm{GaAs}$, существенно уменьшаются, вблизи этих пиков появляются новые пики. Изучение зависимостей $\delta(\varphi)$, снятых при различных $E_{p}$, показали, что при $E_{p} \approx 200 \mathrm{eV}$ глубина выхода ИВЭ составляет $\sim 50 \AA$. При этом на кривых зависимостей $\delta(d)$ пики GaAs полностью исчезают, а интенсивности пиков GaAlAs существенно увеличиваются. По-видимому, при МЛЭ-росте пленка GaAlAs имеет высокую монокристалличность и ее постоянная решетки почти идентична постоянной решетке

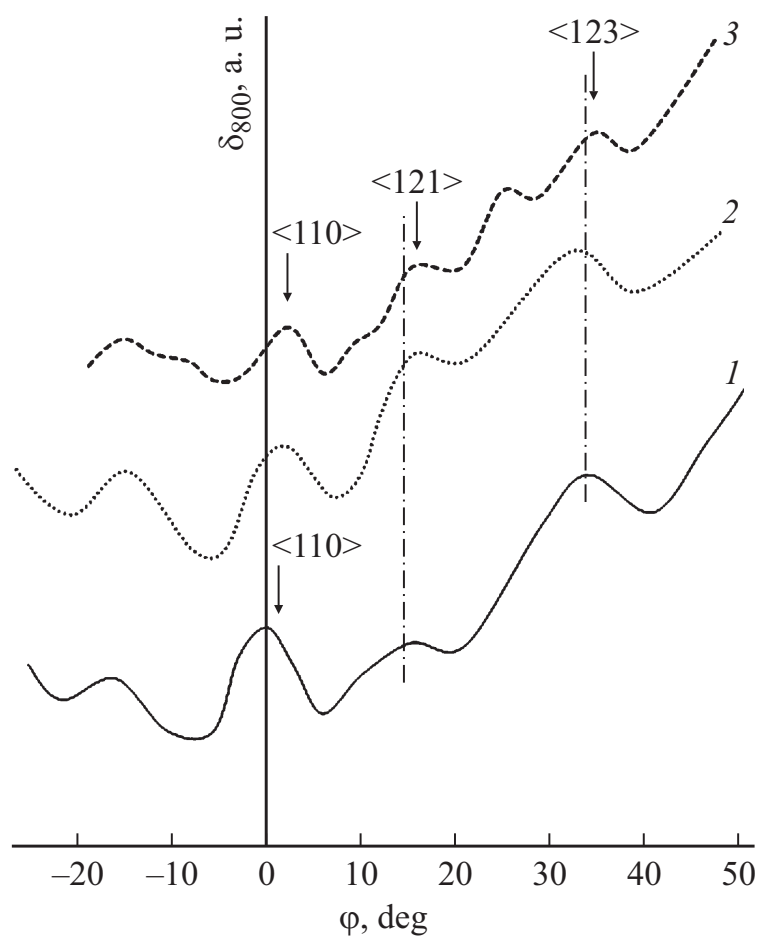

Рис. 2. Зависимости $\delta_{800}(\varphi)$ для чистого GaAs (кривая 1 ) и для систем $\mathrm{GaAs}$ с пленкой $\mathrm{Ga}_{0.5} \mathrm{Al}_{0.5} \mathrm{As}$ толщиной $50 \AA$, полученных методом ионной имплантации (кривая 2) и МЛЭ (кривая 3). 
$\mathrm{GaAs,}$ однако кристаллографические ориентации пленки и подложки совпадают друг с другом не полностью.

Известно, что даже после тщательной очистки $\operatorname{GaAs}(110)$, на его поверхности имеется определенное количество атомов углерода и наблюдается некоторая релаксация поверхности. Все эти факторы могут привести к некоторому изменению кристаллографической ориентации МЛЭ-пленки GaAlAs по отношению к GaAs. В случае ионной имплантации поверхностные слои полностью очищаются от углерода и приповерхностные слои аморфизируются. Толщина аморфизированных слоев $\mathrm{GaAs}$ составляет $300-400 \AA$, что в $3-4$ раза больше, чем толщина ионно-легированных слоев. При прогреве происходит одновременная кристаллизация аморфизированных слоев GaAs и кристаллизация ионноимплантированных слоев GaAs с образованием соединения типа $\mathrm{Ga}_{0.5} \mathrm{Al}_{0.5} \mathrm{As}$. По-видимому, все это способствует формированию трехкомпонентной эпитаксиальной пленки, совпадающей с ориентацией матрицы.

\section{Заключение}

На основе анализа полученных результатов о структуpe, эмиссионных и оптических свойствах тонких пленок GaAlAs/GaAs можно сделать следующие выводы:

1. Зонно-энергетические параметры, эмиссионные свойства и параметры решетки тонких $(d \approx 50-100 \AA)$ пленок GaAlAs, полученных методами МЛЭ и ионной имплантацией, мало отличаются друг от друга. В случае пленок, полученных методом ионной имплантации, кристаллографическая ориентация пленки и подложки хорошо совпадают друг с другом.

2. Глубина зоны выхода ИВЭ и фотоэлектронов для $\mathrm{GaAs}$ составляет $\sim 100-120 \AA$, а для пленки $\mathrm{Ga}_{0.5} \mathrm{Al}_{0.5} \mathrm{As}-150-160 \AA$. Значения коэффициентов $\eta$ соответственно для $\mathrm{GaAs}$ и $\mathrm{Ga}_{0.5} \mathrm{Al}_{0.5} \mathrm{As}$ практически идентичны, а значения коэффициентов ИВЭ $\delta_{m}$ отличаются на $20-25 \%$. Коэффициент отражения света $K$ во всей исследованной области длин волн (как в области поглощения, так и в области максимального отражения) в GaAlAs до двух раз превышал значение $K$ в GaAs. Прогрев системы GaAlAs/GaAs при $T \approx 600^{\circ} \mathrm{C}$ в течение 5-10 $\min$ приводил к некоторому уменьшению $K$ в области интенсивного поглощения света $(\lambda \leq 580 \mathrm{~nm})$. Одной из причин этого может быть наличие в пленках GaAlAs некоторых избыточных атомов Ga или As.

\section{Конфликт интересов}

Авторы заявляют, что у них нет конфликта интересов.

\section{Список литературы}

[1] Эпитаксия и гетероструктуры. / Пер. с англ. Под ред. Ж.И. Алферова, Ю.В. Шмарцева. М.: Мир, 1989. 582 с.

[2] Laref S., Mec-abih S., Abbar B., Bouhafs B., Laref A. // Physica. B. 2007. Vol. 396. P. 169.
[3] Золотарев В.В., Лешко А.Ю., Лютецкий А.В., Николаев Д.Н., Пихтин Н.А., Подоскин А.А., Слипченко С.О., Соколова З.Н., Шамахов В.В., Арсентьев И.Н., Вавилова Л.С., Бахвалов К.В., Тарасов И.С. // ФТП. 2013. Т. 47. Вып. 1. С. 124-128.

[4] Середин П.В., Домашевская Э.П., Арсентьев И.Н., Винокуров Д.А., Станкевич А.Л., Prutskij Т. // ФТП. 2013. Т. 47. Вып. 1. С. 3-8.

[5] Чалдыщев В.В., Школьник А.С., Евтихиев В.П., Holden T. // ФТП. 2006. Т. 40. Вып. 12. С. 1466-1469.

[6] Середин П.В., Глотов А.В., Домашевская Э.П., Арсентьев И.Н., Винокуров Д.А., Станкевич А.Л., Тарасов И.С. // ФТП. 2009. Т. 43. Вып. 12. С. 1654-1661.

[7] Донаев С.Б., Умирзаков Б.Е., Ташмухамедова Д.А. // ЖТФ. 2015. Т. 85. Вып. 10. С. 148-151.

[8] Domashevskaya E.P., Seredin P.V., Lukin A.N., Bityutskaya L.A., Grechkina M.V., Arsent'ev I.N., Vinokurov D.N., Tarasov I.S. // Surf. Interf. Analysis. 2006. Vol. 8. N 4. P. 828.

[9] Домашевская Э.П., Гордиенко Н.Н., Румянцева Н.А., Середин П.В., Агапов Б.Л., Битюикая Л.А., Арсентьев И.Н., Вавилова Л.С., Тарасов И.С. // ФТП. 2008. Т. 42. Вып. 9. C. 1086-1093.

[10] Умирзаков Б.Е., Нормурадов М.Т., Ташмухамедова Д.А., Ташатов А.К. Наноматериалы и перспективы их применения. Ташкент: MERIYUS, 2008. 256 с.

[11] Бронштейн И.М., Фрайман Б.С. Вторичная электронная эмиссия. М.: Наука, 1969. 408 с.

[12] Аброян И.А. // УФН. 1971. Т. 104. Вып. 1. С. 15-50. 